This paper is published in the open archive of Mid Sweden University

DIVA http://miun.diva-portal.org

with permission of the publisher

Citation for the peer-reviewed published paper:

Vidal D, Zou X, Uesaka T. Modelling coating structure development : MonteCarlo deposition of particles with irregular shapes. Nordic Pulp and Paper Research Journal. 2004;19(4):420-427.

URL to article at publishers site:

http://dx.doi.org/10.3183/NPPRJ-2004-19-04-p420-427 


\title{
Modelling coating structure development: Monte-Carlo deposition of particles with irregular shapes
}

\author{
David Vidal, Xuejun Zou and Tetsu Uesaka, Pulp and Paper Research Institute of Canada, Québec, Canada
}

KEYWORDS: Coating, Models, Deposition, Particles, Solids Content, Pigment, Particle size, Particle shape

SUMMARY: There is an increasing interest in enhancing coated paper performance by combining the unique properties of various pigments, i.e. developing multi-pigment coating formulations. An understanding of the synergy between pigments of different sizes and shapes is therefore becoming critical. The literature on this subject is rather empirical and fragmentary. In this report, we introduce an improved 3D Monte-Carlo deposition model to simulate coating structure development for irregularly shaped particles. The model relies on the minimization of the total energy of the particle system, which is defined as a sum of three terms: a pair interaction that accounts for collisional interactions, a gravitational potential energy, and a drag potential energy that takes into account the drainage rate of the colour. The model predicts that pigments with higher aspect ratios give a more porous structure and a smoother surface when applied at high solids content. Coating at low solids content $(<45-55 \%)$ is shown to produce "looser" and more disordered structures, especially in the case of platelet pigments like clay. These results agree with practical and experimental observations. When blending clays with calcium carbonates, the model predicts the occurrence of maximum pore volume and surface micro-roughness at specific mixing ratios. The location of these maxima varies significantly with the particle's aspect ratio, the relative size of the blended pigments, and the solids content of the suspension. With further experimental validation, this model has the potential of being used as a tool for designing and optimizing coating formulations.

ADDRESS OF THE AUTHORS: Pulp and Paper Research Institute of Canada (Paprican), 570 St.-Jean Blvd., PointeClaire, Québec, Canada H9R 3J9.

Corresponding author: David Vidal (dvidal@paprican.ca)

There is within the coating industry a growing interest for blending pigments of different types to achieve specific end-use properties, to improve runnability and to reduce cost. For example, clay and calcium carbonate are sometimes blended to balance runnability, brightness, opacity and print quality of offset printing grades, while silica is blended with clay or calcium carbonate to reduce cost of inkjet coated grades. Understanding the synergy between the pigments is critical for the development of multi-pigment coating formulations. This synergy is mainly controlled by the morphology of the respective pigments, i.e. size and shape.

Although it is well known that particle shape has a big impact on the packing structures (Hagemeyer 1960 Cumberland and Crawford 1987), the literature abounds mainly in numerical and experimental studies about the packing of spheres. The packing of irregular particles is inherently more complex and hence, the numerical and experimental literature on the subject is scarce and fragmentary (Cumberland and Crawford 1987, German
1989). From a numerical standpoint, the complexity comes from the challenges of modelling the particle shape, handling the rotation of the particles and detecting the contact between such irregular particles. Only a few 3-D numerical models have been proposed to study the packing of aspherical particles. Buchalter and Bradley (1994) have performed depositions of 3-D monodispersed spheroids using a Monte-Carlo technique. This approach showed some promise, but the particle collision algorithm they used did not allow the mixing of particles with different sizes and shapes, which limits the practical scope of the model. Coelho and coworkers (1997) developed a 3-D sequential sedimentation model to pack aspherical particles such as spheroids, cylinders and parallelepipeds. However, such a sequential approach does not simulate the fluid-driven consolidation occurring during the coating process. The authors acknowledged that such forced depositions could be better described by the Monte-Carlo approach of Buchalter and Bradley (1994), whose model accounts for the particle hard-core interactions during the deposition process.

In a previous series of articles (Vidal, Zou, and Uesaka 2003) we introduced a 3-D particle-deposition model based on a Monte-Carlo deposition method to simulate coating structure development during coating consolidation. We have demonstrated that this approach is very useful for optimising coating colors in order to achieve the desired end-use performance of coated papers. However, this first version of the model approximated pigments as spheres. Although this approximation is satisfactory for pigments such as $\mathrm{TiO} 2$ and low-aspectratio GCC, it is not realistic for aspherical pigments such as needle-like PCC or platelet clays. Therefore, an improvement of the existing model to take into account particles with irregular shapes is needed in order to understand, predict and optimize multi-pigments coating formulations.

In this report, we will first briefly review the modelling methodology used. Then, we will show the impact of particle shape and size, solids content and pigment blending on coating structures as predicted by the model and compare it with available literature data. Finally, we will present in the Appendix $A$ the improvements added to our original Monte-Carlo deposition model in order to take into account particles with irregular shapes.

\section{Modelling Methodology}

The aim of this section is not to present in detail the modelling methodology, since this has been done in a previous report (Vidal, Zou, and Uesaka 2003). Instead, we will give a very brief description and limit ourselves to highlighting the improvements and the differences with the previous model. More details can be found in the Appendix A. 


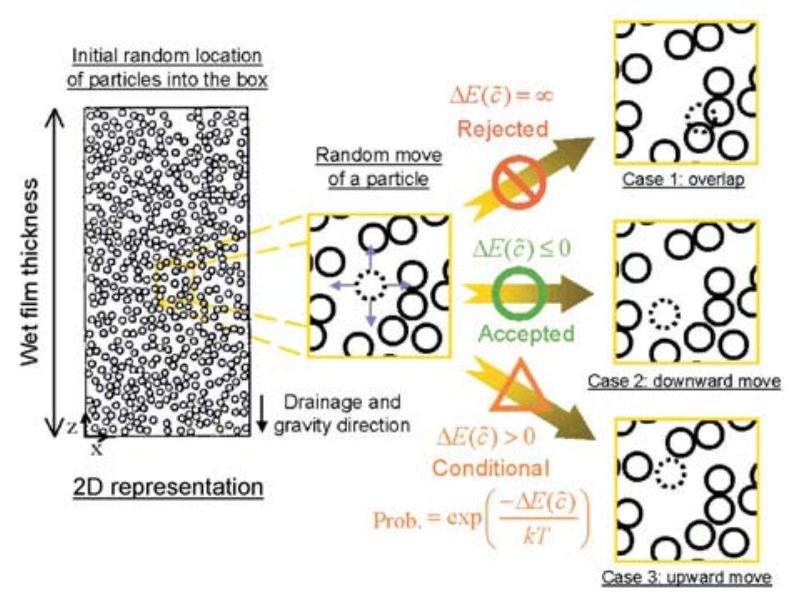

Fig 1. Summary of the Monte-Carlo procedure used.

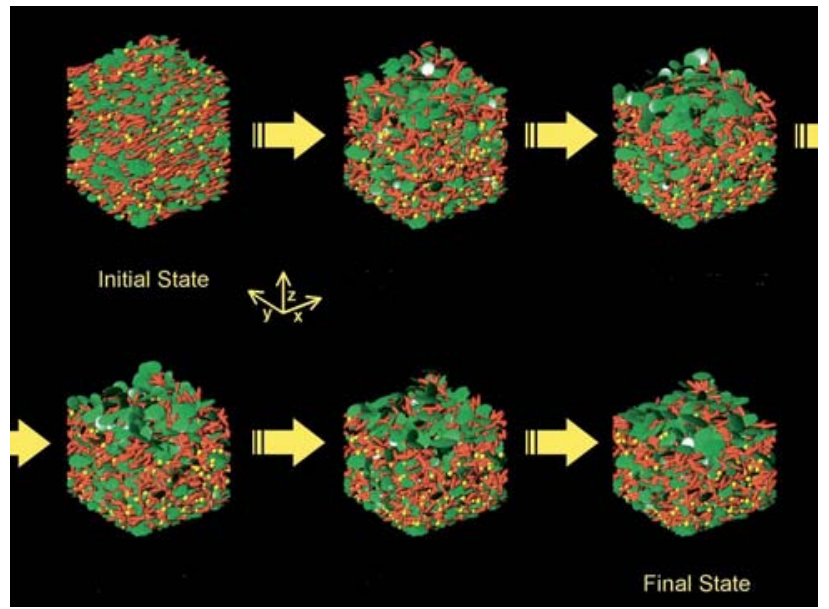

Fig 2. A sequence of states obtained for the deposition of a polydispersed multi-pigment blend using the Monte-Carlo procedure.

The model moves the particles, one at a time, stochastically and iteratively using a Monte-Carlo procedure in a domain representing the wet-film thickness of the coating and having periodic boundary conditions. The individual random particle moves are accepted if the total energy of the system $\mathrm{E}(\tilde{\mathrm{c}})$ is minimized. The latter is defined as a sum of three terms, i.e. the pair interaction $U(\tilde{c})$, the gravitational potential energy $\mathrm{E}_{\mathrm{g}}(\tilde{\mathrm{c}})$ and the drag potential energy $\mathrm{E}_{\mathrm{d}}(\tilde{\mathfrak{c}})$. Fig 1 summarizes this minimization procedure. Particle moves are carried out until the average particle displacement reaches a value lower than a certain percentage (e.g. $0.1 \%$ ) of the initial average particle displacement. As illustrated by Fig 2, this minimization procedure leads to the deposition of the particles under the combined action of gravity and the drag force exerted on each particle by the fluid phase in motion.

After a final packed structure is formed various advanced computer tools for irregularly shaped particles packings need to be used to characterize the pore and surface structures, e.g. in terms of pore volume fraction, surface micro-roughness, pore size distribution, pore surface area, orientational order, etc. A future report will describe in detail the methods used by these tools (Desaulniers, Vidal, and Bertrand 2005), but a short description of the methods can be found in Vidal, Zou, and Uesaka (2003). Here, we will present only results for two of these properties, namely the pore volume fraction (i.e. porosity) and the surface micro-roughness. These two properties control various end-use performances. Pore volume is one of the parameters that control, e.g., ink setting rate, which in return determines print gloss and ink tack. Micro-roughness affects end-use properties such as sheet gloss.

The major improvement of this new model compared to the previous version is the ability to handle aspherical particles through a shape approximation using ellipsoids. More specifically, clay particles are approximated by oblate spheroids and calcium carbonates by prolate spheroids. This implies the implementation of:

- a more advanced overlap detection algorithm for ellipsoids;

- a numerical procedure to compute the drag coefficient of ellipsoidal particles arbitrarily oriented with respect to the fluid flow;

- and a new random displacement procedure to take into account translation but also rotation of the irregularly shaped particles.

Furthermore, in the previous model, two distinct parameters were used to control the drainage rate, namely the average drainage speed $\mathrm{V}$ and the maximum translation distance $\delta$ (see Vidal, Zou, and Uesaka 2003). These two parameters have now been coupled. In the new model, the equivalent of the maximum translation distance, i.e. the maximum kinetic energy constraint $\mathrm{K}_{\max }$ (see Appendix A), is now set as the unique drainage parameter. The average drainage speed is taken as the average particle displacement distance from the previous iteration. This links to some extent the average drainage speed to the specific maximum kinetic energy constraint. As a result, the drainage speed decreases with increasing iteration number, mimicking the decrease of the actual drainage speed due to the formation of the filtercake.

Since its common use in the pigment industry, the concept of aspect ratio (referred as $\phi$ later on) is used throughout this report to characterize particle shape. Ellipsoids used to mimic coating pigments are defined by 3 semi-axes. The aspect ratio is simply the ratio of the longest ellipsoid's semi-axis over its shortest. Hence, for platelet particles, a higher aspect ratio means flatter platelets, whereas for needle-like particles, a higher aspect ratio corresponds to more elongated needles.

Each numerical data point given in this paper is an average of 20 simulations in order to obtain statistically reliable results. The size of the domain used for the simulations was at least 10 times the longest particle's semi-axis. We found that this was sufficient to avoid influence of the domain size on the pore volume fraction. For the initial state before the deposition, we assumed that all the particles were oriented horizontally due to shear forces in the application nip, i.e. the longest particle semi-axis was horizontal. Of course, since coating colors are highly concentrated suspensions the initial orientation can have quite some effect on the dry coating structure obtained. Appendix $B$ presents other 
variables used for the simulations.

In this section, we will study the impact of particle shape and solids content on pore volume and surface micro-roughness. Secondly, we will investigate the effect of blending acicular (i.e. needle-like) and platelet particles on the coating structures and compare our results with available literature data. We recognize that pigment polydispersity has a big impact on coating structures. Although polydispersity can already be handled with the current model, in this first report we will focus on monodispersed pigments for the sake of simplicity and conciseness. A separate report investigating the effects of pigment polydispersity shall be issued in the near future. However, since we are investigating monodispersed pigments, any comparison with experimental data is at this point limited because real pigments are polydispersed by nature.

\section{Results and Discussion}

\section{Effect of Aspect Ratio and Solids Content}

In order to study the effect of particle shape, depositions of mono-sized platelet- and needle-like particles with different aspect ratios have been simulated. In these simulations, the total volume of pigments was kept constant as well as the length of the longest particle's semi-axis. This means that when the aspect ratio is increased, platelets keep the same diameter but become flatter, and needles keep the same length but become thinner. This leads to an increasing number of particles for the same total volume. As we shall see, this has a definite impact on the resulting coating structures.

\section{Platelet particle case}

Fig 3 presents the relationship between the pore volume (i.e. porosity) of the coating layer and the particle aspect ratio for depositions performed at various solids content. Starting from a sphere (aspect ratio equal to one) and increasing the aspect ratio first decreases notably the pore volume up to an aspect ratio around 1.6-2.0 and then the trend is reversed. Buchalter and Bradley (1994) using a similar Monte-Carlo method have noticed the same trend for pore volume vs. particle aspect ratio,

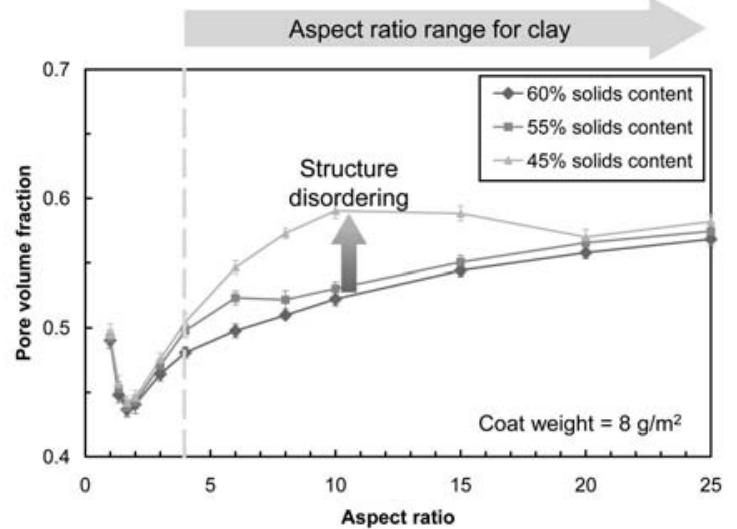

Fig 3. Variation of pore volume fraction with respect to the aspect ratio and the solids content of mono-sized platelet particles (immobilization solids content $\approx 63 \%$ ). although the magnitude of the initial decrease and the subsequent increase were rather different due to modelling differences between the deposition procedures. Furthermore, note that clays have usually an aspect ratio above 4 . So according to Fig 3, for clays of fixed platelet diameter increasing the aspect ratio (i.e. using fine or delaminated clay instead of calcined clay) should always create bulkier structures. This is consistent with practical observations.

Interestingly, the solids content of the suspension has quite an impact on pore volume. As shown in Fig 3, reducing solids content from $60 \%$ to $55 \%$ increases slightly the pore volume fraction. However, when the solids content is further reduced to $45 \%$, the pore volume is significantly increased in aspect ratio range of 5-20. This is attributable to a structure disordering occurring at low solids content as can be seen from simulation visualizations (not presented here). Since the shear under the blade initially orients the particles horizontally, at high enough solids content (i.e. close to the immobilisation solids content - in this case around 63\%) the particles have not enough room to rotate and reorient with the vertical drainage flow. Hence, the packing keeps the ordered structure induced by the shear. Conversely, at low solids content, particles have much more freedom to rotate around during the deposition process. Then, the ordered structure induced by shear is disrupted by the particle reorientation process, especially in the upper part of the packing. However, above a certain aspect ratio (above 20 for the three solids contents studied), the solids content seems to have almost no impact anymore on the pore volume. This means that at these aspect ratios the rotation of particles is hindered by another phenomenon, which is the high level of collisions. As we mentioned in the introduction of this section, when the aspect ratio is increased, the total number of particles also increases. More particles inside the same volume implies a higher probability of collisions between particles, which reduces the freedom of movement and disrupts less the initial orientational ordering.

Fig 4 presents the variation of surface micro-roughness with respect to particle aspect ratio and color solids content. As expected, increasing the aspect ratio generally decreases the surface micro-roughness. But as the solids content is lowered, the same structure disordering observed for the pore volume occurs. We end up actually with an increase of micro-roughness over a range of aspect ratios that depends on the solids content. Finally, as in the case of pore volume, above a certain value of aspect ratio the collisions are so frequent that ordering is preserved.

At this point, one can ask if such particle disordering or reorientation occurs in reality. Some experimental data published in the literature seem to confirm the model prediction. Indeed, Lepoutre (1978) observed that the surface of a coating applied at $40 \%$ solids on an absorbent substrate exhibited a very disordered arrangement of the kaolin plates compared to a coating applied at $60 \%$. However, the model may overestimate this particle reorientation. For example, long polymer chains like starch or CMC that can form network structures with pigments, colloidal interactions (not taken into account 


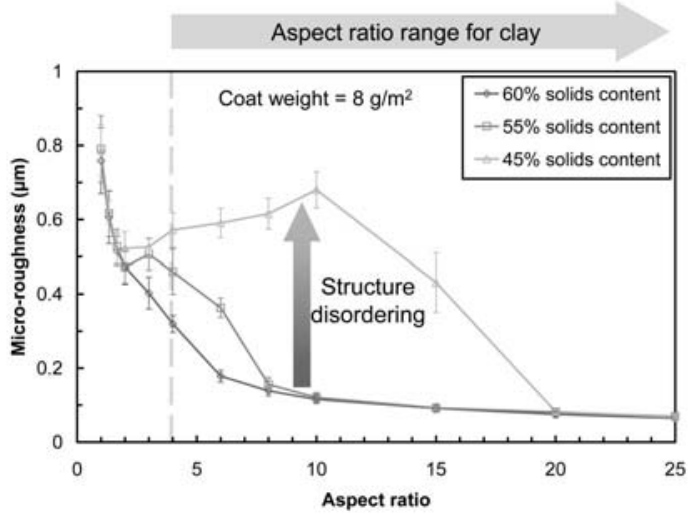

Fig 4. Variation of surface micro-roughness with respect to the aspect ratio and the solids content of mono-sized platelet particles (immobilization solids content $\approx 63 \%$ ).

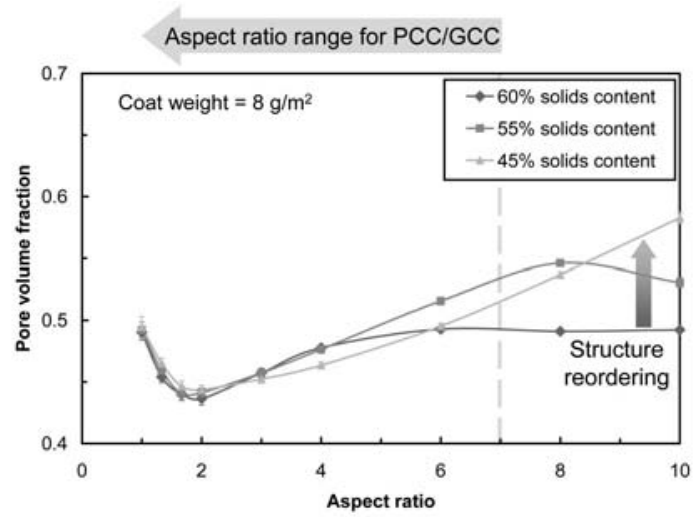

Fig 5. Variation of pore volume fraction with respect to the aspect ratio and the solids content of mono-sized acicular particles (immobilization solids content $\approx 63 \%$ ).

by the model yet) and pigment polydispersity may hinder the reorientation to a certain extent.

\section{Acicular particle case}

Similarly to platelet particles, the pore volume of acicular particle packings starts first to decrease when the particle aspect ratio is increased up to 1.6-2.0 (Fig 5). Further increase of the aspect ratio results in an increase of the pore volume. This trend has also been predicted to a certain extent by Buchalter and Bradley's simulations (1994). Coating pigments like GCC and PCC have aspect ratios varying usually between 1 and 7 . Within this range we can see that, the effect of solids content on pore volume is rather limited. However, outside this range, the pore volume seems to reach a plateau at high solids content whereas it still increases at low solids content. As shown by simulation visualizations (not presented here), a complete reordering of particles occurs at low solids content for very elongated acicular particles, especially in the upper layer of the coating. As in the case of platelet particles, low solids contents allow particles to freely rotate and reorient with the drainage flow, but in this case the acicular particles fall preferentially along their major semi-axis which allows them to find a different ordered state instead of disorder. However, note that the new structure has a rougher micro-surface due to the new particle orientation and a higher pore volume due to a more disordered bottom layer.

As shown by Fig 6, increasing the aspect ratio of

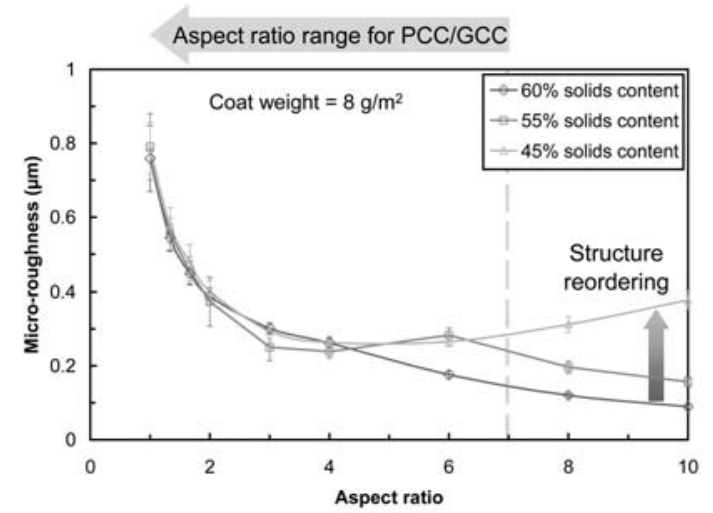

Fig 6. Variation of surface micro-roughness with respect to the aspect ratio and the solids content of mono-sized acicular particles (immobilization solids content $\approx 63 \%$ ).

acicular particles reduces significantly the surface microroughness. Again, solids content seems to have only a slight effect on the range of interest for coating pigments (1 to 7). However, at high aspect ratio (6.0-7.0 and upper), "structure reordering" produces an increase in surface micro-roughness.

Until now, we found no data in the literature that could confirm the occurrence of this structure reordering for needle-like particles. Of course, the presence of aggregates of needles could strongly influence this result. However, by initially orienting acicular particles in the same direction, we assume that any needle aggregates have been destroyed in the nip application due to shear and do not have time to reform due to colloidal interactions during the deposition. As in the case of platelet particles, the particle reorientation could be hindered to a certain extent by the presence of long polymer chains, colloidal interactions and polydispersity. However, this reordering seems to occur for very elongated acicular particles, i.e. well beyond the aspect ratios usually encountered for coating pigments. This may explain why this has not been observed.

\section{Effect of Pigment Blending}

In order to study the effect of pigment blending, depositions of monodispersed platelet- and needle-like particles with different aspect ratios have been performed. Platelet particles mimic clay and acicular/rhombic particles represents PCC or GCC depending on the aspect ratio. In these simulations, the individual particle volume has been kept constant when the aspect ratio is changed. Clay particles have a volume 1.6 times bigger than acicular/rhombic particles.

\section{Blending monodispersed platelet particles with various} monodispersed acicular/rhombic particles

Platelet particles of aspect ratio of 10 have been blended with various acicular particles at different mixing ratios and at $50 \%$ solids content. Fig 7 presents the variation of pore volume with volume-based mixing ratio for the various cases. The location of the maximum pore volume is predicted and varies with the needle's aspect ratio. The maximum pore volume is obtained for mixing ratios varying between $30 / 70$ and $50 / 50$ depending on the calcium carbonate blended. These results can be qualitatively compared with the experimental data obtained by 


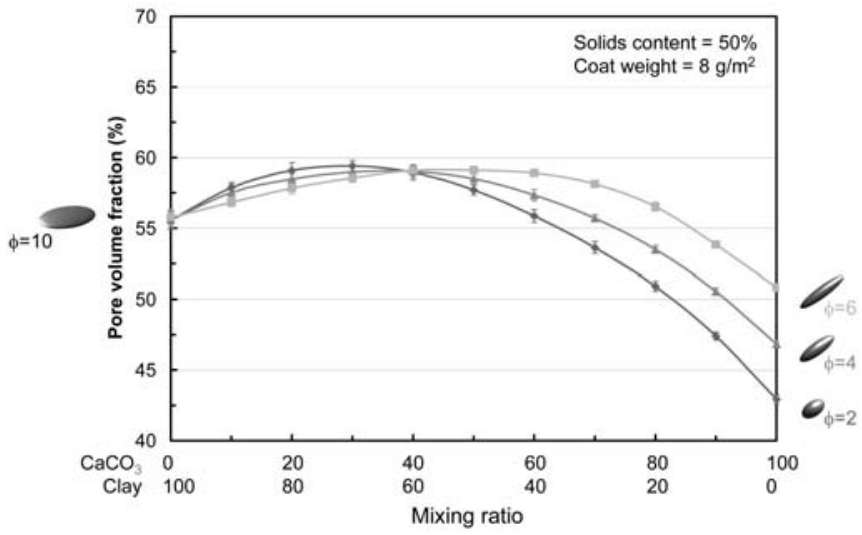

Fig 7. Variation of pore volume for binary blends of monodispersed clay and calcium carbonates with various aspect ratios.

Hagemeyer (1960). Hagemeyer prepared various blends of polydispersed platelet clay and polydispersed acicular and rhombic calcium carbonates with 50\% solids content. The suspensions were centrifuged for $1 \mathrm{hr}$. at $2000 \mathrm{rpm}$ and the centrifuged volume (proportional to pore volume) was determined. Fig 8 presents Hagemeyer's experimental results. The trends obtained are quite similar to those of Fig 7. But a direct comparison is not possible since the simulations are performed with monodispersed pigment blends whereas the experiments used polydispersed coating pigments of unknown particle size and shape distributions. Both simulations and experiments predict a lower pore volume for rhombic or acicular calcium carbonate packings than for clay packings. They also predict that the mixing ratio that gives the maximum pore volume depends on the particle shapes.

Fig 9 presents the variation of surface micro-roughness for the various blends. For blends of pigments with $\phi=2$ or $\phi=4$, adding a small amount of calcium carbonate in a clay coating or, conversely, adding a small amount of clay in a calcium carbonate coating always increases the micro-roughness. For $\phi=6$, the micro-roughness is initially reduced when a small amount of calcium carbonate is added, then increases again before reaching a maximum. In any case, the location of the maximum micro-roughness depends significantly on the shape of the needle-like particles.

Blending monodispersed acicular particles with various monodispersed platelet particles

Figs 10 and 11 present, respectively, the variation of pore volume and surface micro-roughness for blends of monodispersed calcium carbonate with various monodispersed clays. When the clay aspect ratio is increased, the location of the maximum pore volume does not change much, but the overall pore volume curve is shifted towards higher pore volume fractions. Incidentally, the overall surface micro-roughness is reduced and the maximum moves towards the $100 \%$ calcium carbonate formulation.

\section{Effect of solids content on pigment blending}

In the previous sub-section, we have investigated the effects of pigment blending at a fixed (50\%) solids content. However, as we can see in Fig 12, solids content has a significant impact on the magnitude and the location of the maximum pore volume, with lower solids content giving a higher porosity and a maximum location

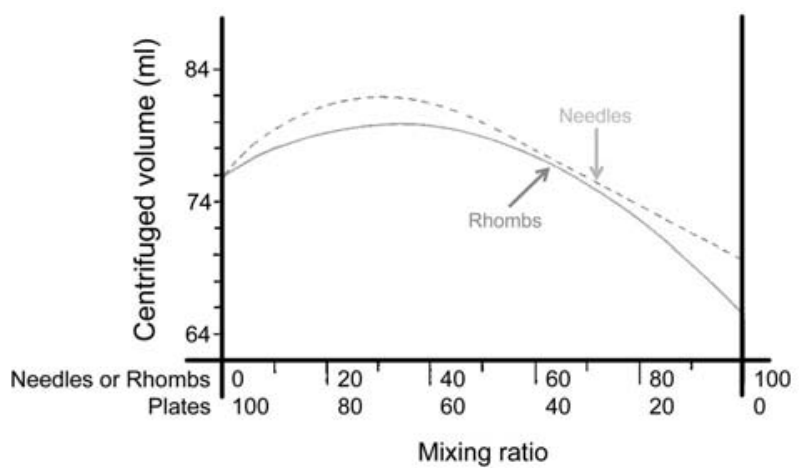

Fig 8. Experimental variation of pore volume when blending polydispersed platelet clay with polydispersed acicular and rhombic calcium carbonates (after Hagemeyer 1960)

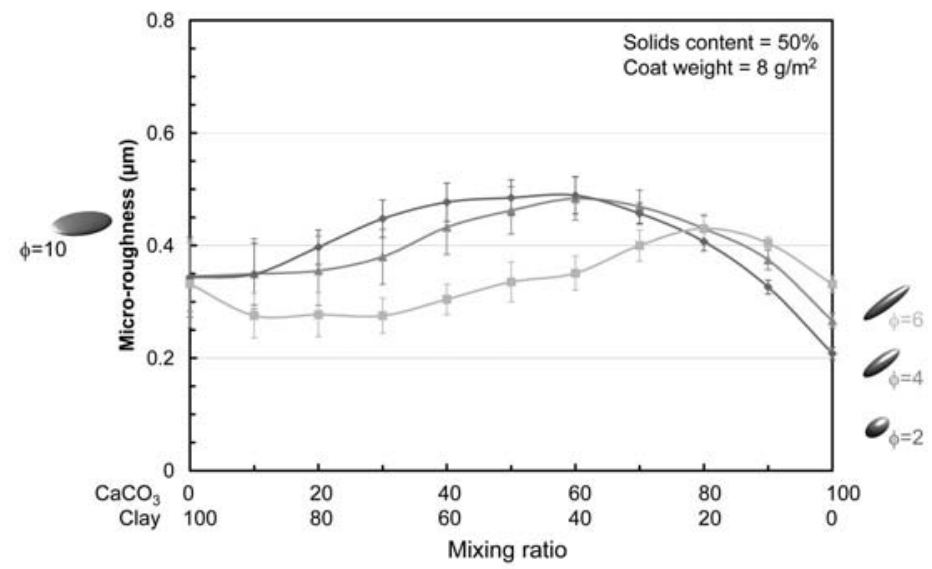

Fig 9. Variation of micro-roughness for binary blends of mono dispersed clay and calcium carbonates with various aspect ratios.

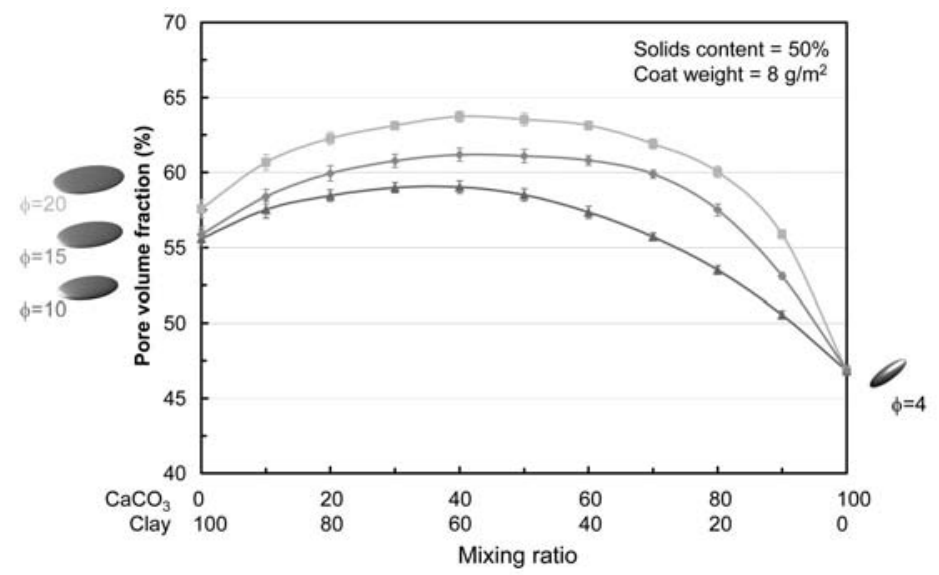

Fig 10. Variation of pore volume for binary blends of monodispersed calcium carbonate and clays with various aspect ratios.

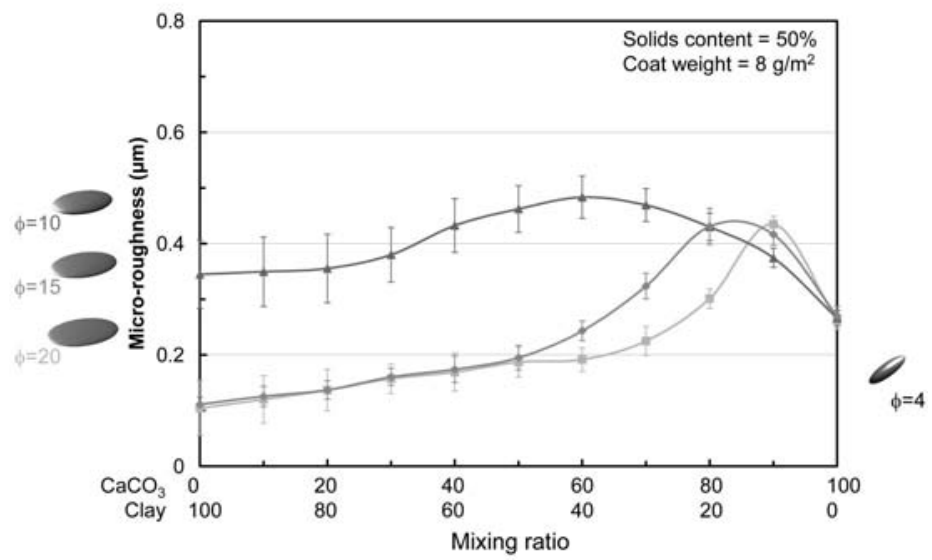

Fig 11. Variation of micro-roughness for binary blends of monodispersed calcium carbonate and clays with various aspect ratios. 


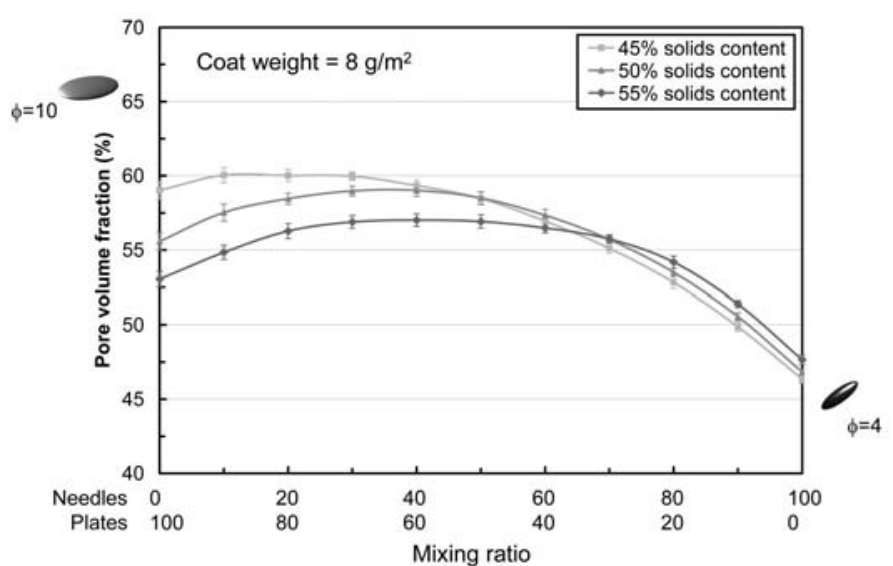

Fig 12. Effect of solids content on pore volume of a binary pigment blend.

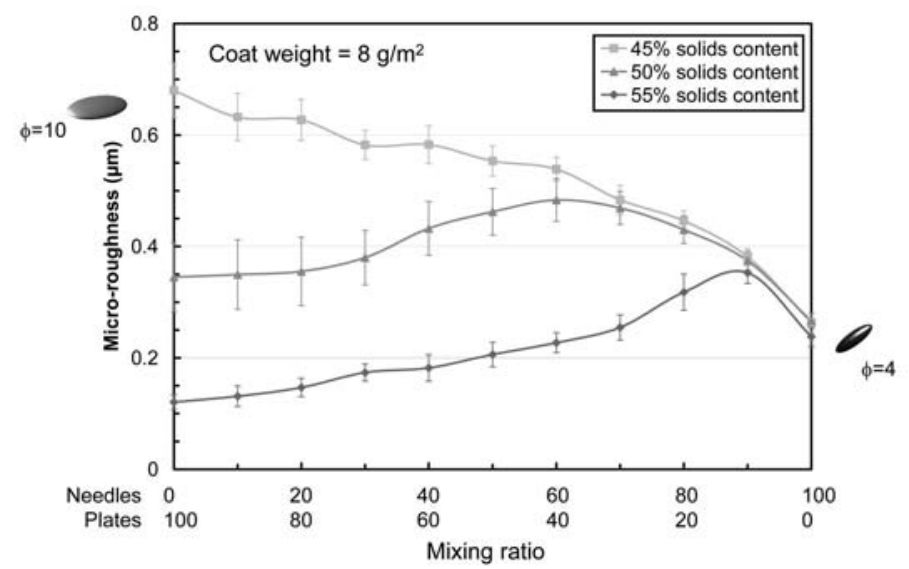

Fig 13. Effect of solids content on surface micro-roughness of a binary pigment blend.

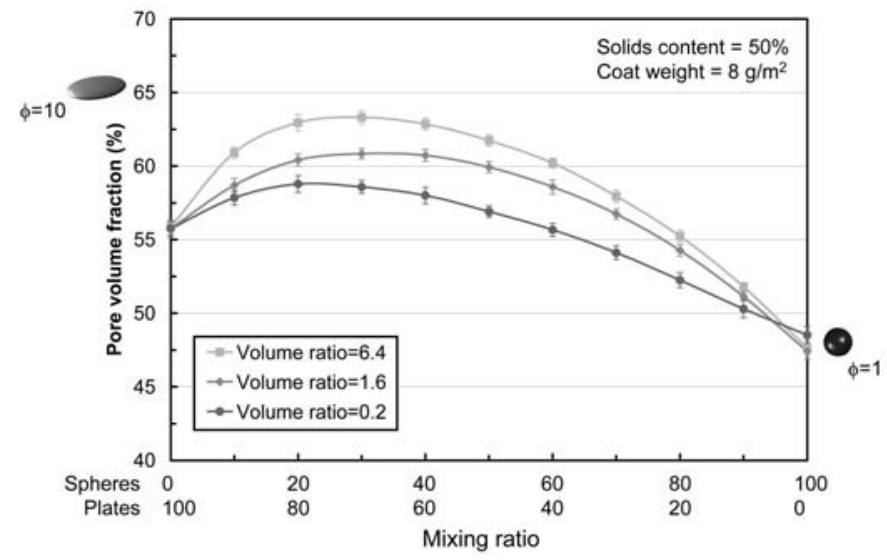

Fig 14. Effect of particle volume ratio on the pore volume relationship for a binary blend of platelets and spheres (platelet's longest semi-axis $=0.5 \mu \mathrm{m}$ ).

closer to $100 \%$ clay formulation. This stems from the disordering of the structure formed by platelet particles when solids content is lowered, as mentioned in the previous section. In addition, the maximum surface micro-roughness increases drastically and also shifts towards the $100 \%$ clay formulation (Fig 13).

Effect of relative particle size on pigment blending Until now throughout this report, we looked at the effect of particle shape. However, in pigment blending, the relative particle size between the different types of pigments blended has also its importance and should not be disregarded. In Fig 14, platelet particles are mixed with spherical particles of different sizes. Increasing the particle volume ratio between platelets and spheres (i.e. $\mathrm{V}_{\text {platele }} / \mathrm{V}_{\text {sphere }}$ ) shifts significantly the overall relationship towards higher pore volume fraction and changes slightly the location of the maximum.

The impact of particle size in monodispersed pigment blending gives us a glimpse of the impact of pigment polydispersity on coating structures. Thus, we believe that the relationships drawn in this report should not be too much extrapolated to the general case of polydispersed blends. As we mentioned previously, since the complexity of the phenomena and the computations involved with polydispersity, it will be investigated separately in a future report. Although the actual code can handle pigment polydispersity, the implementation of a fully parallel code on a powerful parallel computer will be required in order to tackle realistic situations, i.e. wide particle size distributions.

\section{Conclusions}

- The existing 3D Monte-Carlo deposition model for spherical particles is improved to simulate coating structure development of irregularly shaped particles.

- The model predicts that the use of pigments with higher aspect ratio (especially above 2) gives a more porous structure and a smoother surface when applied at high solids contents. This agrees with practical observations.

- Lowering solids content $(<45-55 \%$ for an immobilisation solids content at $63 \%$ ) affects significantly the coating structures through particle reorientation due to the fluid phase flow. For clay pigments, low solids contents create a more porous and rougher coating layer due to particle disordering. For acicular or rhombic calcium carbonate, the overall effect of solid content is small for the aspect ratios of interest (1 to 7) because of the inherent capability of such particles for efficient packing.

- When blending clay pigments with calcium carbonate pigments, the model predicts the occurrence of maximum pore volume and surface micro-roughness at specific mixing ratios. The location of these maxima varies significantly with the particle's aspect ratio, the solids content of the suspension and the relative size of the pigments in the blend. These results agree qualitatively with published experimental data. They also underline the need for considering the pigment polydispersity in order to predict blending relationships more accurately.

\section{Acknowledgements}

The authors would like to thank Evelyne Desaulniers and François Bertrand from École Polytechnique for providing us with numerical characterization tools that helped us to determine pore volume and surface micro-roughness of packings of irregularly shaped particles, as well as for having graciously offered us their computer resources and for stimulating discussions. Also special thanks go to Dionissios Hristopulos for useful discussions and his close review of the manuscript. 


\section{APPENDIX A — Model Improvements}

This appendix presents the improvements implemented in the original model (Vidal, D., Zou, X. and Uesaka, T. 2003) in order to take into account aspherical particles such as coating pigments.

\section{Overlap Detection Algorithm for Ellipsoids}

In the case of spheres, the overlap detection algorithm is quite simple since it consists of determining the distance between the centres of two spheres. The spheres do not overlap when the distance is greater than the sum of their radii. However, detection of overlap is much more challenging in the case of ellipsoids. A sphere is characterized by one degree of freedom, i.e. its radius, whereas an ellipsoid has 6 degrees of freedom, i.e. its three semiaxes and its three angles of rotation. The efficiency of the whole deposition model relies on the speed of the overlap detection algorithm. A few overlap detection algorithms for ellipsoids have been reported in the literature (Vieillard-Baron 1972, Rimon and Boyd 1997, Perram and Wertheim 1985). Perram and Wertheim's algorithm appeared to be the most efficient for our application. It relies on the resolution of maximization problem of the contact function $\mathrm{F}$ :

$$
\max _{0 \leq 5 \leq 1 \leq 1} F(\lambda)\left\{\begin{array}{c}
<1 \\
=1 \\
>1
\end{array}\right\} \text { if ellipsoids A and } B\left\{\begin{array}{c}
\text { overlap } \\
\text { are externally tangent } \\
\text { do not overlap }
\end{array}\right\}
$$

where $\lambda$ is an unknown Lagrange multiplier in the interval $0 \leq \lambda \leq 1$. The reader is referred to the article of Perram and Wertheim (1985) for a complete definition of this contact function. Finding the maximum of $F(\lambda)$ requires the use of an iteration procedure known as Brent's method which combines root bracketing (i.e. $0 \leq \lambda \leq 1$ ), bisection technique and inverse quadratic interpolation. This method is especially dedicated to minimization problems in which the derivatives of the function to minimize are not (easily) available. The advantage of this method is that the detection of non-overlaps (which are much more frequent than overlaps) does not require the maximum to be found, i.e. as soon as a value $F(\lambda)>1$ is found, the non-overlap is guaranteed and the iterative search can be broken off. Moreover, the authors report that an initial guess of $\lambda=1 / 2$ resulted in $F(\lambda)>1$ for over $90 \%$ of non-overlaps. This makes the algorithm quite efficient.

\section{Drag Coefficient for Ellipsoids}

The drag potential energy in our deposition model is defined as follows:

$$
E_{d}(\tilde{c})=\sum_{j=1}^{N} C_{j} \mu V z_{j}
$$

where $\mu$ is the fluid phase viscosity, $\mathrm{V}$ the average drainage speed, and $C_{j}$ and $z_{j}$ are respectively the drag coefficient and the vertical location of the $\mathrm{j}$-th particle. In the case of spherical particles, the drag coefficient is equal to $6 \pi r_{j}$ where $r_{j}$ is the radius of the sphere. The drag coefficient for an ellipsoidal particle is more complicated since it depends on the ellipsoid's semi-axes and its orientation with regard to the flow. If we assume that the flow points in the $\mathrm{z}$ direction, the overall drag coefficient for an arbitrary oriented ellipsoid can be expressed as:

$$
C_{j}=\left(C_{j, a}\left(z \cdot e_{a}\right) e_{a}+C_{j, b}\left(z \cdot e_{b}\right) e_{b}+C_{j, c}\left(z \cdot e_{c}\right) e_{c}\right) \cdot z
$$

where $\mathbf{e}_{a}, \mathbf{e}_{b}$ and $\mathbf{e}_{\mathrm{c}}$ are the unit vectors of the ellipsoid's semi-axes, and $\mathrm{C}_{\mathrm{j}, \mathrm{a}}, \mathrm{C}_{\mathrm{i}, \mathrm{b}}$ and $\mathrm{C}_{\mathrm{j}, \mathrm{c}}$ are the principal translational resistances of the $\mathrm{j}$-th ellipsoid respectively with $a_{j}$, $b_{j}$ and $c_{j}$ semi-axes (Happel and Brenner 1965). The calculation of the principal translational resistances is explained in the next section.

\section{Calculation of the Principal Translational Resistances for Ellipsoids}

$\mathrm{C}_{\mathrm{j}, \mathrm{a}}$ is given by the following equations (similarly for $\mathrm{C}_{\mathrm{j}, \mathrm{b}}$ and $\mathrm{C}_{\mathrm{j}, \mathrm{c}}$ ) (Happel and Brenner 1965):

$$
\mathrm{C}_{\mathrm{j}, \mathrm{a}}=6 \pi \mathrm{R}_{\mathrm{j}, \mathrm{a}}
$$

with

$$
\mathrm{R}_{\mathrm{j}, \mathrm{a}}=\frac{8}{3\left(\chi+\psi_{\mathrm{a}} \mathrm{a}_{\mathrm{j}}{ }^{2}\right)}
$$

and

$$
\begin{aligned}
& \chi=\int_{0}^{\infty} \frac{\mathrm{d} \lambda}{\Delta} \\
& \psi_{\mathrm{a}}=\int_{0}^{\infty} \frac{\mathrm{d} \lambda}{\left(\mathrm{a}_{\mathrm{j}}^{2}+\lambda\right) \Delta} \\
& \Delta=\sqrt{\left(\mathrm{a}_{\mathrm{j}}{ }^{2}+\lambda\right)\left(\mathrm{b}_{\mathrm{j}}{ }^{2}+\lambda\right)\left(\mathrm{c}_{\mathrm{j}}{ }^{2}+\lambda\right)}
\end{aligned}
$$

Integrals in Eqs (A6) and (A7) cannot be expressed explicitly. These integrals are known as elliptic integrals of the first and second kind, respectively, and numerical algorithms have been developed by Carlson to evaluate them (Press, Teukolsky, Vetterling, and Flannery 1994), Let's now call $R_{F}$ and $R_{D}$ the results obtained from those algorithms, then we have:

$$
\begin{aligned}
& \chi=2 \mathrm{R}_{\mathrm{F}}\left(\mathrm{a}_{\mathrm{j}}{ }^{2}, \mathrm{~b}_{\mathrm{j}}{ }^{2}, \mathrm{c}_{\mathrm{j}}{ }^{2}\right) \\
& \psi_{\mathrm{a}}=\frac{2}{3} \mathrm{R}_{\mathrm{D}}\left(\mathrm{c}_{\mathrm{j}}{ }^{2}, \mathrm{~b}_{\mathrm{j}}{ }^{2}, \mathrm{a}_{\mathrm{j}}{ }^{2}\right)
\end{aligned}
$$

Then, $E q(A 4)$ becomes:

$$
C_{j, a}=\frac{8 \pi}{\left(R_{F}\left(a_{j}{ }^{2}, b_{j}{ }^{2}, c_{j}{ }^{2}\right)+\frac{a_{j}{ }^{2}}{3} R_{D}\left(c_{j}{ }^{2}, b_{j}{ }^{2}, a_{j}{ }^{2}\right)\right)}
$$

Hence, to compute the drag coefficient, only Carlson's elliptic integral algorithms and Eqs (A3) and (A11) are required. Finally, note that the principal translational resistances actually need only to be computed once and for all.

\section{Random Displacement Procedure for Ellipsoids}

In the previous model, the spherical particles were moved randomly one at a time within a small neighbourhood characterized by a maximum translation distance $\delta$ that can also be seen as a maximum kinetic energy constraint. In the case of ellipsoids, both translation and rotation need to be considered. Hence, the new trial position for the $\mathrm{j}$-th ellipsoidal particle is given by: 
$x_{j}^{\prime}=x_{j}+\Delta x_{j}$

$\mathrm{y}_{\mathrm{j}}^{\prime}=\mathrm{y}_{\mathrm{j}}+\Delta \mathrm{y}_{\mathrm{j}}$

$z_{j}^{\prime}=z_{j}+\Delta z_{j}$

$\alpha_{j}^{\prime}=\alpha_{j}+\Delta \alpha_{j}$

$\beta_{j}^{\prime}=\beta_{j}+\Delta \beta_{j}$

$\gamma_{j}^{\prime}=\gamma_{j}+\Delta \gamma_{j}$

[A12]

where $x_{j}, y_{j}, z_{j}$ represent the location of the $j$-th particle, $\alpha_{\mathrm{j}}, \beta_{\mathrm{j}}, \gamma_{\mathrm{j}}$ are the rotation angles of the ellipsoid's semiaxes $a_{j}, b_{j}$ and $c_{j}$, respectively, with respect to the global coordinate frame.

In the previous model, the vector $(\mathrm{V}, \delta)$ served as the drainage rate parameter. In the current model, a unique drainage rate parameter is established. Firstly, the notion of maximum translation distance $\delta$ is replaced by a maximum kinetic energy constraint called $\mathrm{K}_{\max }$. It sets the maximum range of the "small neighbourhood" within which particles can freely translate and rotate per iteration. Secondly, V is calculated at each iteration from the average particle move generated at the previous iteration for a given $\mathrm{K}_{\max }$. Therefore, $\mathrm{K}_{\max }$ and $\mathrm{V}$ are unambiguously related.

Two separate kinetic energy constraints could have actually been used for translation and rotation, but this could lead, for example, to cases where particles excessively rotate with respect to their translation. In reality, the fluid phase imparts a certain energy to the particles through the drag force. This energy is converted into both translational and rotational kinetic energy or dissipated through collisions. Hence, to mimic more realistically the physics of the deposition process, we couple translation and rotation by means of a unique kinetic energy constraint, i.e. $\mathrm{K}_{\max }$. The actual kinetic energy constraint $\mathrm{K}_{\mathrm{j}}$ for each particle move varies. Its value is set by multiplying the maximum kinetic energy constraint with a random number $(0 \leq \zeta \leq 1)$ :

$$
\mathrm{K}_{\mathrm{j}}=\zeta \mathrm{K}_{\max }
$$

The coupling between rotation and translation is expressed by the following equation, which is based on a kinetic energy balance:

$$
K_{j}=\zeta K_{\max }=\Delta x_{j}^{2}+\Delta y_{j}^{2}+\Delta z_{j}^{2}+\frac{I_{j, a}}{v_{j}} \Delta \alpha_{j}^{2}+\frac{I_{j, b}}{v_{j}} \Delta \beta_{j}^{2}+\frac{I_{j, c}}{v_{j}} \Delta \gamma_{j}^{2}[A 14]
$$

where $I_{j, a}, I_{j, b}$ and $I_{j, c}$ are the moments of inertia with respect to the corresponding ellipsoid's semi-axes, and $\mathrm{v}_{\mathrm{j}}$ represents the particle volume. For an ellipsoid, $\mathrm{I}_{\mathrm{j}, \mathrm{a}}$ (similarly for $I_{j, b}$ and $I_{j, c}$ ) is given by:

$$
I_{j, a}=\frac{1}{5} v_{j}\left(b_{j}^{2}+c_{j}^{2}\right)
$$

Finally, we need to distribute the kinetic energy constraint among the three translations $\left(\Delta \mathrm{x}_{\mathrm{j}}, \Delta \mathrm{y}_{\mathrm{j}}, \Delta \mathrm{z}_{\mathrm{j}}\right)$ and the three rotations $\left(\Delta \alpha_{\mathrm{j}}, \Delta \beta_{\mathrm{j}}, \Delta \gamma_{\mathrm{j}}\right)$. This is achieved by means of six uniformly distributed random numbers $\left(-1 \leq \xi_{\mathrm{x}}, \xi_{\mathrm{y}}\right.$, $\left.\xi_{z}, \xi_{\alpha}, \xi_{\beta}, \xi_{y} \leq 1\right)$. To satisfy $E q(A 14)$, we chose:

$$
\begin{aligned}
& \Delta \mathrm{x}_{\mathrm{j}}=\frac{\xi_{\mathrm{x}}}{\left|\xi_{\mathrm{x}}\right|} \sqrt{\frac{\left|\xi_{\mathrm{x}}\right|}{\sum_{i}\left|\xi_{i}\right|} \sqrt{\zeta \mathrm{K}_{\max }}} \\
& \Delta \mathrm{y}_{\mathrm{j}}=\frac{\xi_{\mathrm{y}}}{\left|\xi_{y}\right|} \sqrt{\frac{\left|\xi_{\mathrm{y}}\right|}{\sum_{i}\left|\xi_{i}\right|}} \sqrt{\zeta \mathrm{K}_{\max }} \\
& \Delta \mathrm{z}_{\mathrm{j}}=\frac{\xi_{z}}{\left|\xi_{z}\right|} \sqrt{\frac{\left|\xi_{z}\right|}{\sum_{i}\left|\xi_{i}\right|}} \sqrt{\zeta \mathrm{K}_{\max }}
\end{aligned}
$$

$$
\begin{aligned}
& \Delta \alpha_{j}=\frac{\xi_{a}}{\left|\xi_{a}\right|} \sqrt{\frac{\left|\xi_{a}\right|}{\sum_{i}\left|\xi_{i}\right|}} \sqrt{\frac{v_{j} \zeta K_{\max }}{I_{j, a}}} \\
& \Delta \beta_{j}=\frac{\xi_{p}}{\left|\xi_{p}\right|} \sqrt{\frac{\left|\xi_{p}\right|}{\sum_{i}\left|\xi_{i}\right|}} \sqrt{\frac{v_{j} \zeta K_{\max }}{I_{j, b}}} \\
& \Delta \gamma_{j}=\frac{\xi_{y}}{\left|\xi_{\eta}\right|} \sqrt{\frac{\left|\xi_{i}\right|}{\sum_{i}\left|\xi_{i}\right|}} \sqrt{\frac{v_{j} \zeta \mathrm{K}_{\max }}{\mathrm{I}_{j, c}}}
\end{aligned}
$$

where $i=x, y, z, \alpha, \beta, \gamma$.

\section{APPENDIX B - Variables used for the simulations}

\begin{tabular}{lc}
\hline $\begin{array}{l}\text { TABLE BI } \\
\text { Variables used for the simulations. }\end{array}$ \\
\hline Fluid phase viscosity $(\mathrm{Pa} . \mathrm{s})$ & 0.01 \\
Maximum kinetic energy constraint $\left(\mathrm{m}^{2}\right)$ & $1.10^{-14}$ \\
Temperature $(\mathrm{K})$ & $0^{*}$ \\
Density of the pigments $\left(\mathrm{kg} / \mathrm{m}^{3}\right)$ & 2500 \\
Density of the soluble binder $\left(\mathrm{kg} / \mathrm{m}^{3}\right)$ & 1500 \\
Soluble binder content $(\mathrm{pph})$ & 7 \\
\hline
\end{tabular}

* The Brownian motion has been neglected for these simulations.

\section{Literature}

Buchalter, B.J. and Bradley, R.M. (1994): "Orientational Order in Amorphous Packings of Ellipsoids", Europhys. Lett., 26(3),159.

Coelho, D., Thovert, J.-F. and Adler, P.M. (1997): "Geometrical and Transport Properties of Random Packings of Spheres and Aspherical Particles", Phys. Rev. E, 55(2), 1959.

Cumberland, D.J. and Crawford, R.J. (1987): "The Packing of Particles", Elsevier Science, Amsterdam, The Netherlands, $151 \mathrm{p}$.

Desaulniers, E., Vidal, D. and Bertrand, F. (2005): "Characterization of Packings of Particles with Irregular Shapes", to be published.

Dobkin, D.P. and Kirkpatrick, D.G. (1983): "Fast Detection of Polyhedral Intersection", Theor. Comp. Sci., 27, 241.

German, R.M. (1989): "Particle Packing Characteristics", Metal Powder Industries Federation, Princeton, NJ, USA, 443 p.

Hagemeyer, R.W. (1960): "The Effect of Pigment Combination and Solids Concentration on Particle Packing and Coated Paper Characteristics", Tappi J., 43(3), 277.

Happel, J. and Brenner, H. (1965): "Low Reynolds Number Hydrodynamics with Special Applications to Particulate Media", Prentice-Hall, Englewood Cliffs, NJ, USA. Lepoutre, P. (1978): "Paper Coatings: Substrate Absorbency and Coating Structure", Tappi J., 61(5), 51.

Perram, J.W. and Wertheim, M.S. (1985): "Statistical Mechanics of Hard Ellipsoids. I. Overlap Algorithm and the Contact Function”, J. Comp. Phys., 58, 409. Press, W.H., Teukolsky, S.A., Vetterling, W.T., and Flannery, B.P. (1994), "Numerical Recipes in Fortran - The Art of Scientific Computing", 2nd ed., Cambridge University Press, Cambridge, UK.

Rimon, E. and Boyd, S.P. (1997): "Obstacle Collision Detection Using Best Ellipsoid Fit”, J. Int. Rob. Syst., 18, 105.

Vidal, D., Zou, X. and Uesaka, T. (2003) "Modeling Coating Structure Development Using A Monte-Carlo Deposition Method. Part I Modeling Methodology", Tappi J., 2(4), 3-7.

Vidal, D., Zou, X. and Uesaka, T. (2003): "Modeling Coating Structure Development Using A Monte-Carlo Deposition Method. Part II Validation and Case Study", Tappi J., 2(5), 16-20.

Vieillard-Baron, J. (1972): "Phase Transitions of the Classical Hard-Ellipse System", J. Chem. Phys., 56, 4729.

Manuscript received March 10, 2004 Accepted June, 2004 